\title{
Single-Channel Permeability from \\ Markovian Milestoning, Umbrella Sampling, and Voltage Simulations
}

\author{
Yi-Chun Lin, Yun Lyna Luo* \\ Department of Pharmaceutical Sciences, Western University of Health Sciences, California, \\ USA
}

Corresponding email:

luoy@westernu.edu

\begin{abstract}
Permeations of ions and small molecules through membrane channels have diverse functions within cells. Various all-atom molecular dynamics (MD) simulations methods have been developed for computing free energy and crossing rate of permeants. However, a systemic comparison across different methods is scarce. Here, using a carbon nanotube as a model of small conductance ( $\sim 2 \mathrm{pS}$ ) ion channel, we systemically compared three classes of MD-based approaches for computing single-channel permeability for potassium ion: equilibrium free energy-based approach using umbrella sampling, rare-even sampling approach using Markovian milestoning, and steady-state approach using applied voltages. The consistent kinetic results from all three methods demonstrated the robustness of MD-based methods in computing ion channel permeation. Two solvent boundary conditions are tested for milestoning and yield consistent forward and backward mean first passage time (MFPT). The advantages and disadvantages of each method are discussed, with the focus on the future applications of milestoning in more complex systems.
\end{abstract}




\section{Introduction}

Ion channels are complex biological nanopores that perform vital physiological functions with high sensitivity and precision. Molecular dynamics (MD) simulation is a powerful tool for calculating the functional properties of ion channels directly from their dynamic structures. Various MD-based methods have been developed for investigating the thermodynamics (freeenergy differences between states) and kinetics (rate constants, barriers-crossing) of ions/small molecules (aka. solute) permeation at the single-channel level. Pioneer all-atom MD simulations on ion channels have focused on computing ion permeation from equilibrium free energy profiles or potential of mean force (PMF) in conjunction with electro-diffusion theory ${ }^{1-4}$. The increased performance of all-atom MD simulation codes has also made it possible to simulate singlechannel conduction explicitly under an electrical driving force, either by applying an external electric field ${ }^{5,6}$ or by asymmetric ionic concentration ${ }^{7,8}$. More recently, rare-event sampling strategies have been used to extract the kinetic properties of ion or small-molecule permeation through ion channels directly from MD trajectory without the assumption of electro-diffusion or applying external driving forces. Examples of such applications on ion channels are Weighted ensemble sampling ${ }^{9}$, Markov state models ${ }^{10-13}$, and Milestoning ${ }^{14-16}$.

The aforementioned MD-based approaches have significantly deepened our understanding of the ion channel permeation process at high temporal and spatial resolution. However, a systemic comparison among them is scarce. In this work, we use a carbon nanotube (CNT) as a model (Figure 1a) of small conductance ( $2 \mathrm{pS}$ ) ion channel and compared $\mathrm{K}^{+}$ permeability from milestoning, umbrella sampling, and voltage simulations. Single-channel permeability for a particular ion or molecule is an intrinsic property of each channel, independent of concentration or transmembrane potential (i.e., voltage). It is hence an ideal quantity for rigorous comparison between different experimental and/or computational methods. This CNT system has been used to compute $\mathrm{K}^{+}$permeability using a transition path approach similar to the reactive flux method ${ }^{17}$. We chose this system because its free energy barrier height ( $\left.4 \mathrm{kcal} / \mathrm{mol}\right)$ and microsecond-timescale crossing rate are physiologically relevant. Such a system requires nontrivial sampling (beyond the capability of brute-force MD), but the rigidity of the CNT still allows good convergence and unambiguous comparison of all three methods tested. 
The original milestoning simulation requires running short trajectories in each milestone until they reach another milestone ${ }^{18}$. Here, we make use of the "soft-walls" Voronoi-tessellated Markovian milestoning, which confines the sampling within the Voronoi cells using flat-bottom harmonic restraining potentials ${ }^{19}$. The implementation of this "soft-walls" version (referred to as milestoning thereafter) resembles, to a large degree, the conventional umbrella sampling setup. A detailed comparison of the sampling, PMF, and MFPT results from milestoning and umbrella sampling is the focus of this study. In addition, both cylindrical and spherical bulk boundary conditions are tested for the milestoning method. The CNT system chosen here is designed to satisfy the symmetric single barrier requirement, thus allowing us to check the robustness of milestoning method by computing both inward and outward permeation rates. We also show that the MFPT from milestoning is extremely sensitive to the frequency of recording the MD trajectories (frame stride). The consistent results obtained from three methods, summarized in Table 1, demonstrated the robustness of MD simulation in capturing single-channel permeability. The advantage of milestoning for future applications on more complex systems is discussed.

\section{Theory and Methods}

\section{Relation between single-channel permeability, MFPT, and conductance}

Assuming permeating solutes do not interact under sufficient low concentration, single-channel permeability $\left(\mathrm{cm}^{3} / \mathrm{s}\right)$ can be related linearly to the rate of crossing $k$ or mean first passage time (MFPT) $\langle t\rangle$ under equilibrium $P=k / c=1 / c\langle t\rangle$, in which $c$ is the symmetric solute concentrations. Here, we use the number of molecules per second (num/s) for $k, \mathrm{~s} /$ num for $\langle t\rangle$, and num $/ \mathrm{cm}^{3}$ for $c$. This relation can be applied to the charged or neutral solute. For ionic permeation under voltage and/or concentration gradient, Goldman-Hodgkin-Katz (GHK) flux equation describes the ionic flux across a homogenous membrane as a function of a constant electric field (voltage) and an ionic concentrations gradient. When applied to a membraneembedded single-channel model and ions only cross the membrane through the channel, $P$ in the GHK equation corresponds to the single-channel permeability. GHK flux equation thus relates 
single-channel conductance $\gamma$, a nonequilibrium property, to the equilibrium property $P$. Notice that the crossing rate $k, \operatorname{MFPT}\langle t\rangle$, and conductance $\gamma$ are all concentration-dependent; only $P$ is independent of concentration.

\section{System setup and equilibrium protocols}

The coordinates of the carbon tube (CNT) were taken from ref ${ }^{17}$. Briefly, it is an uncapped armchair CNT with $13.5 \AA$ in length and $5.4 \AA$ in radius. Two carbon sheets form an artificial membrane to separate the solution. Constraints were applied to all carbon atoms to keep the system rigid. CHARMM36 force field was used ${ }^{20,21}$. After solvation, the box size was $38 \times 38 \times 75$ $\AA^{3}$, which contained 2503 TIP3P water molecules, six $\mathrm{K}^{+}$and six $\mathrm{Cl}^{-}$. All MD simulations were performed using $1 \mathrm{fs}$ time step using NAMD2.13 package under NVT ensemble, with 1 atm and $300 \mathrm{~K}$ temperature using Langevin thermostat ${ }^{22,23}$. Cutoff for calculating vdW interaction and short-range electrostatic interaction was set at $12 \AA$ and force-switched at $10 \AA$. Long-range electrostatic interactions were calculated using the particle mesh Ewald algorithm ${ }^{24}$. The system was equilibrium for 100ns before conducting umbrella sampling, milestoning and voltage simulations.

\section{Umbrella sampling simulations}

A total of 28 windows $(-27 \AA<z<+27 \AA$ ) were sampled. Each window was separated by $2 \AA$ apart. The tagged $\mathrm{K}^{+}$was restraint by a harmonic restraint on $\mathrm{z}$ and a flat-bottom harmonic cylindrical restraint. The force constant for harmonic restraint was $2.5 \mathrm{kcal} / \mathrm{mol} / \AA$ and cylindrical restraint was $10 \mathrm{kcal} / \mathrm{mol} / \AA$ within $6 \AA$ on $\mathrm{X}-\mathrm{Y}$ radius plane. The reference dummy atom to pull the $\mathrm{K}^{+}$was set at $(0,0,0)$. The harmonic distance restraint was determined by the projected vector along $z$ between the dummy atom and tagged $\mathrm{K}^{+}$. The cylinder restraint was determined by the center of mass of all carbon atoms from CNT. Each window ran with $40 \mathrm{~ns}$. The PMF was computed using pymbar $3.0 .3^{25,26}$. The output frequency was 0.5 ps per frame.

\section{Position-dependent diffusion coefficient $D(z)$}


$\mathrm{D}(\mathrm{z})$ of $\mathrm{K}^{+}$inside the CNT was calculated from umbrella sampling trajectories. The correlation time

$i$ is first extracted from each umbrella window $i$ using $\tau_{i}=\int_{0}^{\infty} \frac{\langle\delta z(t) \delta z(0)\rangle_{i}}{\langle\delta z\rangle_{i}^{2}} d t$ where $\delta z(t)=$ $z(t)-\langle z\rangle$, is the deviation of the $z$-position of the ion at time $t, z(t)$, from the time-averaged position $\langle z\rangle$ in each window. $\left\langle\delta z^{2}\right\rangle_{i}=\left\langle z^{2}\right\rangle_{i}-\langle z\rangle_{i}^{2}$, is the variance. Following the formulation of ref $^{27-29}$., the Laplace transformation of the autocorrelation function of the velocity $z$ in the harmonically restrained umbrella sampling gives $D\left(z_{i}\right)=\frac{\left\langle\delta z^{2}\right\rangle}{\tau_{i}}$.

\section{Markovian Milestoning}

Same as in umbrella sampling, the z-coordinate of the tagged ion was used to define a set of Voronoi cells along the channel pore, and to identify the milestones as the boundaries between the cells. We then ran many local simulations confined in the cells, and we collected the kinetics of transitions between milestones. More specifically, let us introduce a set of $M$ Voronoi cells $B_{\mathrm{i}}$, $i=1, \ldots, M . \mid \mathrm{If}_{j} k_{j \rightarrow i}$ is the rate of attempted escape from cells $B_{\mathrm{i}}$ to $B_{\mathrm{j}}$, since at statistical equilibrium the total flux in and out of each cell is zero, the equilibrium probability $\pi_{\mathrm{i}}$ for the permeant to be in cell $B_{\text {i }}$ satisfies a balance equation:

$$
\sum_{j=1, j \neq i} \pi_{j} k_{j \rightarrow i}=\sum_{j=1, j \neq i} \pi_{i} k_{i \rightarrow j}, \sum_{i=1} \pi_{i}=1 \quad \text { Eq. } 1
$$

The free energy of each cell can be obtained from the solution of Eq. 1 as $-k_{B} T \ln \left(\pi_{\mathrm{i}}\right)$. By defining a milestone $S_{\mathrm{ij}}$ as the boundary between two adjacent Voronoi cells $B_{\mathrm{i}}$ and $B_{j}$, the dynamics of the system is reduced to that of a Markov chain in the state space of the milestone indices ${ }^{4}$. The MFPT between any pair of milestones $S_{\mathrm{ij}}$ and $S_{\mathrm{ik}}$ can hence be calculated from the rate matrix whose elements $q_{i j, i k}$, the rate of moving from milestone $S_{\mathrm{ij}}$ to $S_{\mathrm{ik}}$, are given by Eq. 2:

$$
q_{i j, i k}=\frac{\pi_{i} n_{i j, i k}^{i}}{\pi_{i} r_{i j}^{i}+\pi_{j} r_{i j}^{j}}
$$

where $n_{i j, i k}^{i}$ is the number of transitions from $S_{\mathrm{ij}}$ to $S_{\mathrm{ik}}$, normalized by the time spent in cell $B_{\mathrm{i}}$, and $r_{i j}^{i}$ is the time passed in cell $B_{\mathrm{i}}$ after having hit $S_{\mathrm{ij}}$ before hitting any other milestone, 
normalized by the total time spent in cell $B_{\mathrm{i}}$. The inward and outward MFPT profiles were obtained by reversing the milestone indices when constructing the rate matrix.

Milestoning had same number of Voronoi cells and cell size as Umbrella sampling method. The only difference is that a flat-bottom harmonic restraint of force constant $100 \mathrm{kcal} / \mathrm{mol} / \AA$, instead of the weak harmonic restraint, was used to confine the sampling within each cell. The total sampling time of all 28 cells was $4.9 \mu \mathrm{s}$. The NAMD Colvars output frequency was $0.5 \mathrm{ps}$ and the trajectory frequency was 5 ps per frame. The PMF and MFPT were computed using a set of in-house python scripts https://github.com/yichunlin79/CNT milestoning method with different frame sizes. To check whether the frame size affected the MFPT, additional milestoning simulation was conducted with the output frequency of $0.2 \mathrm{ps}$. The total sampling time of $0.2 \mathrm{ps}$ trajectories was $2.74 \mu \mathrm{s}$. Frame size of 1 ps and 2 ps data were generated from 5 ps frame size trajectory.

\section{Voltage simulations}

After $100 \mathrm{~ns}$ equilibrium simulation, the voltage systems were also run with +/-0.4 $\mathrm{V}$ using NAMD2.13. To be consistent with the umbrella sampling and milestoning, a cylinder restraint was applied to a tagged $\mathrm{K}^{+}$within $6 \AA$ radius and $10 \mathrm{kcal} / \mathrm{mol} / \AA$ for the force constant. All other ions moved freely beyond the cylinder restraint region. The time step was $1 \mathrm{fs}$ and the output frequency was 0.5 ps. Each voltage was run for three replicas of 200 ns.

\section{Results and Discussion}

\section{Permeability computed from inhomogeneous solubility-diffusion equation (ISD)}

For ion or small-molecule permeation, the PMF approach can be generally applied to any solute (neutral or charged) and can be used to compute crossing rates. If the PMF is dominated by a single large barrier and solute diffusion is constant at the barrier region, crossing rate can be estimated via Kramer's theory or transition state theory borrowed from reaction kinetics. Although it is applicable to the CNT model here, for most of the biological ion channels, reaction kinetic approaches may not be applicable. Alternatively, solute permeation may be considered 
as a one-dimensional nonreactive diffusive process that can be described using the fluctuationdissipation theorem. For instance, PMF can be used together with position-dependent diffusion coefficient to estimate permeability using inhomogeneous solubility-diffusion (ISD) equation ${ }^{30}$. ISD equation has been applied successfully in studying solute permeation across membrane 31,32 and can be related to the mean first passage time (MFPT) ${ }^{33}$.

Solute (i.e., ions and small molecules) permeation through ion channel can be described by ISD if the one-dimensional free energy and diffusion along channel normal is sufficient to describe the diffusion process (relaxation of orthogonal degrees of freedom is fast relative to the reaction coordinate) and the solute velocity relaxation time is instantaneous. The only difference with the ISD equation used for membrane permeation is that a flat-bottom lateral potential $u(x, y)$ is often used to confine a single tagged ion in a cylindrical bulk region outside the ion channel. The effective cross-sectional area due to the lateral restraint is thus $\iint e^{-\beta u(x, y)} d x d y$, which can be approximated to $\pi r^{2}$, where $r$ is the radius of the cylinder. Hence, $\pi r^{2}$ defines the effective bulk concentration in the simulated region, which led to the probability of the ion inside the channel $p(z)$ over the true bulk density $\rho$ to be $\frac{p(z)}{\rho}=\pi r^{2} e^{-\beta w(z)}$, in which $w(z)$ is the PMF with the bulk value set to zero at the cylindrical region and $\beta=1 / k_{B} T^{34,35} . T$ is the temperature; $k_{B}$ is the Boltzmann's constant. Therefore, at low ionic concentration, singlechannel permeability can be estimated using a slightly modified ISD equation (Eq 3),

$$
P=\pi r^{2}\left(\int_{z_{1}}^{z_{2}} \frac{e^{\beta w(z)}}{D(z)} d z\right)^{-1} \quad \text { Eq } 3
$$

$\mathrm{D}(\mathrm{z})$ is the position-dependent diffusion constant of the studied permeant along $\mathrm{z}$-axis. The interval of the integration $[z 1, z 2]$ are the lower and upper boundaries of the channel pore, beyond which PMF reaches bulk value. $r$ is the radius of the cylindrical restraint when ion is outside of the $[z 1, z 2]$ interval. It is necessary to set $r$ to be larger than maximum pore radius, so it has no energetic contribution inside the pore. The volume of the cylindrical restraint defines the effective bulk concentration, thus the offset of the bulk PMF value.

In both umbrella sampling and milestoning, same cylindrical restraint with $r=6 \AA$ is applied in bulk region and same window size of $2 \AA$ was used. The only difference is that a 
harmonic restraint with force constant of $2.5 \mathrm{kcal} / \mathrm{mol}$ is applied for all umbrella windows, but a flat-bottom harmonic restraint with force constant of $100 \mathrm{kcal} / \mathrm{mol}$ is applied for all milestoning cells. Figure $\mathbf{1 b}$ and Figure $\mathbf{2} \mathbf{b}$ illustrated the biased sampling distribution imposed by two methods. The PMFs from US are shown in Figure 1c. With bulk value offset to zero, a broad energy barrier of $3.8 \mathrm{kcal} / \mathrm{mol}$ is located inside CNT region, , consistent with a previously reported $\mathrm{PMF}^{17}$. Using the $w(z)$ in Figure $1 \mathrm{c}$ and $\mathrm{D}(\mathrm{z})$ in Figure $\mathbf{1} \mathbf{d}$, the permeability estimated from Eq.1 is $(8.96 \pm 0.02) \times 10^{-16} \mathrm{~cm}^{3} / \mathrm{s}$. From Eq.1, one can also plot local resistant (the integrand of Eq. 1 ) for permeating $\mathrm{K}^{+}$through CNT (Figure 1e) and the integration of the permeation resistance, $1 / P$, as a function of $z$ (Figure 1f). It is not surprising that the $1 / P(z)$ bares the same feature as the MFPT in Figure 2d, although they have different units. 
bioRxiv preprint doi: $\mathrm{https} / / /$ doi.org/10.1101/2021.11.20.469392. this version posted November 20, 2021. The copyright holder for this preprint (which was not certified by peer review) is the author/funder, who has granted bioRxiv a license to display the preprint in perpetuity. It is made available under aCC-BY-ND 4.0 International license.
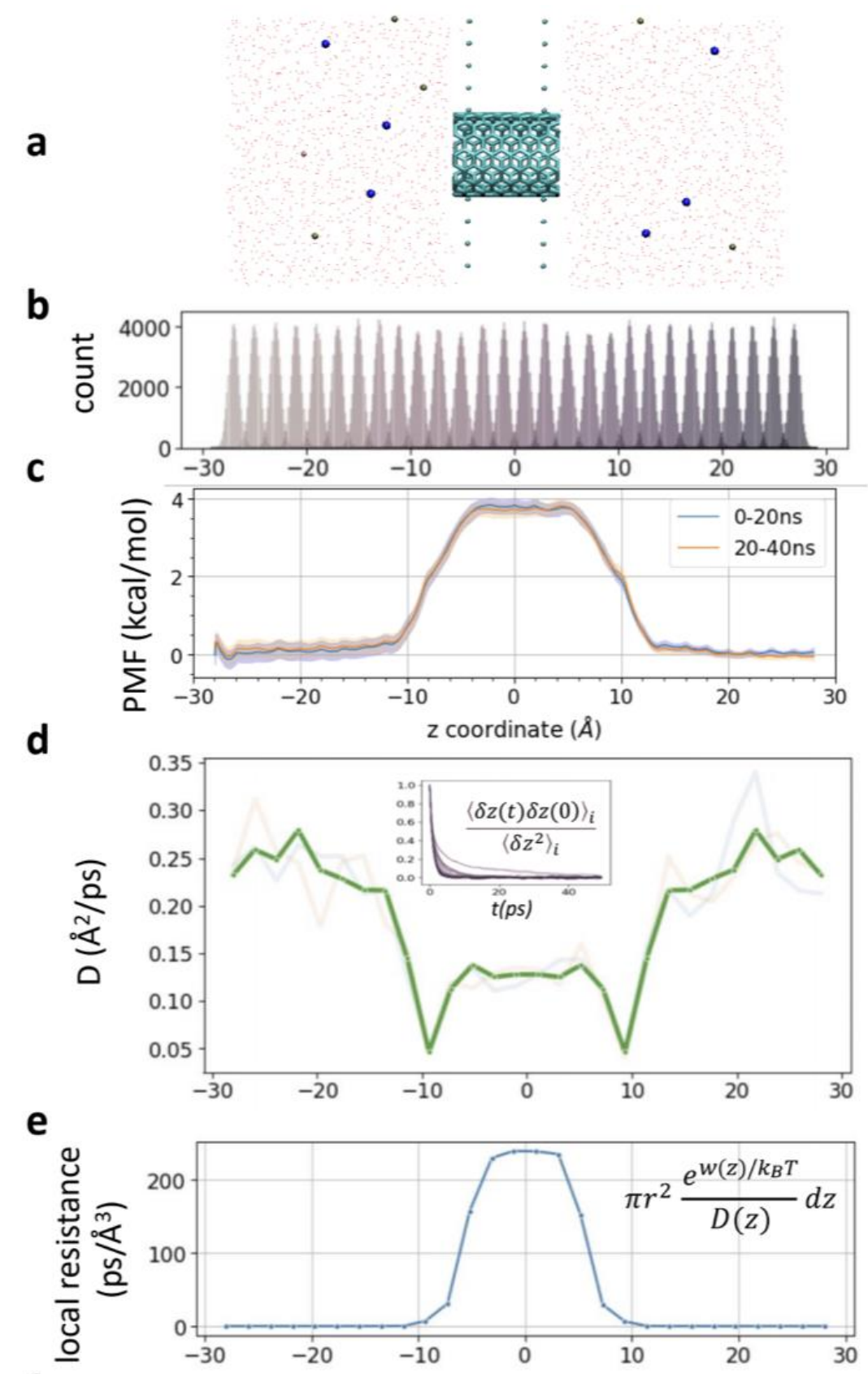

f

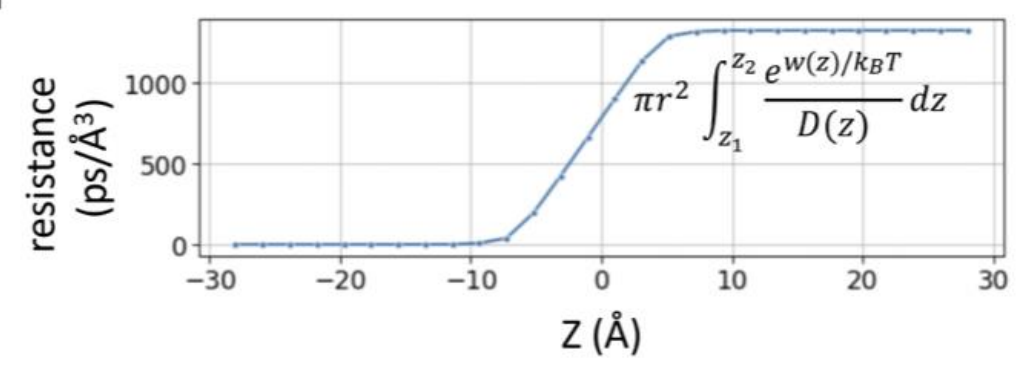


Figure 1 a. Simulated CNT system, consisting of two fixed layer of carbon atoms as water impermeable membrane. $6 \mathrm{~K}^{+}$and $6 \mathrm{Cl}^{-}$ions are shown in tan, blue and pink color dots, CNT in cyan color and water oxygen in red. b. Raw data distribution from 28 umbrella sampling windows (corresponding data from milestoning is shown in Figure 2b). c. PMFs from two blocks of umbrella sampling trajectories, generated from MBAR with 800 bins number and 80,000 data points per window. The PMFs computed from the first 20ns and second 20ns per window are shown with error bars in shaded color. d. Position-dependent diffusion constant of $\mathrm{K}^{+}$computed from umbrella sampling. The transparent lines represent data from the first $20 n$ s and second $20 n s$ per window. Thick green line represents averaged and symmetrized value. Inset is the plot of correlation function for all windows used to compute correlation time $i$ and $\mathrm{D}(\mathrm{z})$ (see methods). e. Local resistant (the integrand of Eq 3 ) for permeating $K^{+}$. f. Integration of the permeation resistance, $1 / P$, as a function of $z$.

\section{Permeability computed from mean first passage time (MFPT)}

The MFPT of a single $\mathrm{K}^{+}$crossing the CNT is computed from Voronoi-tessellated Markovian milestoning simulations (see Methods). The sampling of the tagged $\mathrm{K}^{+}$confined in each $2 \AA$ cell by flat bottom harmonic restraint are shown in Figure $\mathbf{2 a}$ and $\mathbf{2 b}$. Milestoning simulation yields a consistent PMF profile with highest energy barrier of $4.1 \mathrm{kcal} / \mathrm{mol}$ at center of the CNT (Figure 2c). Since the CNT used here is symmetric, a rigorous check of sampling convergence is the perfect symmetricity (mirror image) of the inward and outward MFPT profiles (Figure 2d). The inward and outward MFPT profiles can be obtained by reversing the milestone indices when constructing the rate matrix. We show that while PMF is not sensitive to the frame size, MFPT is highly dependent on the frame size. In this system, frame size above 0.5 ps over-estimates the MPFT due to the missing transition events. Larger frame size also yields less amount of the data, which led to the asymmetric MFPTs. For example, MFPT of 5 ps frame size has ten times less data point than $0.5 p s$ frame size; therefore, it has difficulty to reach convergence even with $18 \mu$ s of milestoning simulations. The maximum frame size will depend on the local diffusion and the shape of the underlying free energy landscape in each system. Frame size $0.2 \mathrm{ps}$ and 0.5 ps yield identical and symmetric MFPT of $2.6 \pm 0.03 \mu \mathrm{s}$ for $\mathrm{K}^{+}$permeation through a barrier of $4.1 \mathrm{kcal} / \mathrm{mol}$.

Using the PMF $w(z)$ and MFPT $\langle t\rangle$ from the milestoning (0.5 ps frame size), the single channel permeability can be computed from Eq. $4^{33}$, 


$$
P=\frac{\pi r^{2} \int_{z 1}^{z 2} e^{-\beta w(z)} d z}{2\langle t\rangle}
$$

Eq 4.

The resulting permeability from the Eq. 4 is $2.92 \times 10^{-16} \mathrm{~cm}^{3} \mathrm{~s}^{-1}$, in good agreement with the permeability of $8.96 \times 10^{-16} \mathrm{~cm}^{3} / \mathrm{s}$ computed from ISD equation above using umbrella sampling data.

Vanden-Eijnden et al. have shown that Markovian Milestoning yields exact MFPTs if the milestones are chosen such that successive transitions between them are statistically independent $^{36,37}$ and thus no definition of lag time is needed. To check this assumption for transitions between two neighboring milestones, in each cell the maximum and minimum waiting time the transition between two neighboring milestones is extracted and plotted in Figure $2 \mathbf{e}$ and $\mathbf{2 f}$. Two plots are almost mirror images because the transition down the slope of PMF is the fastest and against the slope is the slowest. Thus, the longest waiting time $94 \mathrm{ps}$ and the shortest waiting time 9 ps are in the same cell where the PMF is steepest. The velocity decorrelation time is less than the smallest frame size (0.2 ps). The z-position decorrelation time for the tagged $\mathrm{K}^{+}$in each cell are also plotted in Figure $\mathbf{2 g}$. The maximum decorrelation time $7.6 \mathrm{ps}$ is also located at the steepest PMF region. For all pairs of milestones, the z-position decorrelation time is less than the minimum waiting time for successive transition between milestones, thus satisfy the requirement of Markovian Milestoning. 
bioRxiv preprint doi: $\mathrm{https}$ //doi.org/10.1101/2021.11.20.469392. this version posted November 20,2021 The copyright holder for this preprint (which was not certified by peer review) is the author/funder, who has granted bioRxiv a license to display the preprint in perpetuity. It is made available under aCC-BY-ND 4.0 International license.

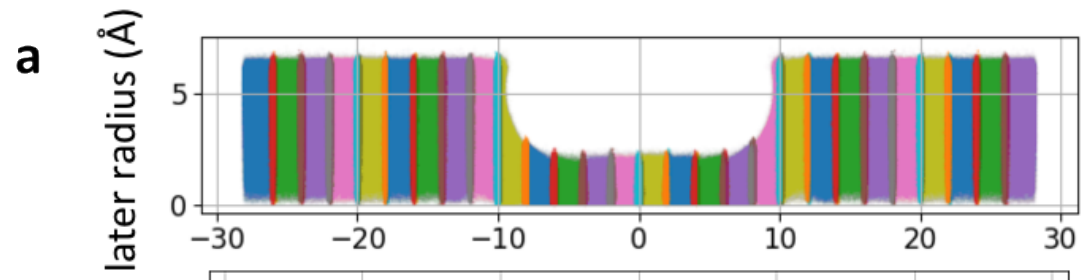

b
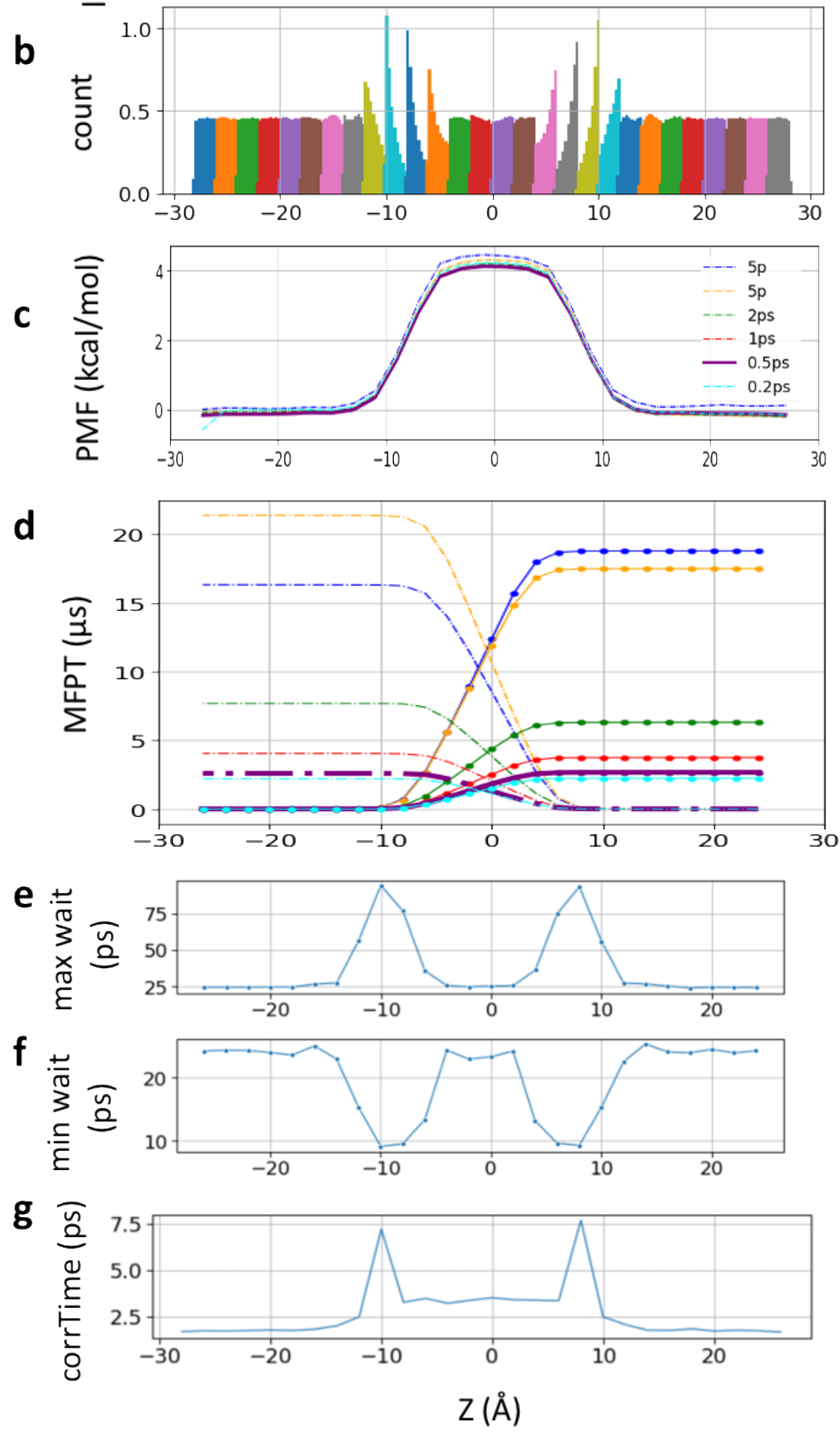
Figure 2 a. Raw data plotted along the channel z-axis and radial distance $R=\sqrt{x^{2}+y^{2}}$ from channel center axis $(x, y=0,0)$ from 28 milestoning sampling cells. b. Distribution of the milestoning data along z-axis (same plot from US is shown in Fig1a). c. PMF from Milestoning sampling. Different plot color represents different frame size. From large to small frame size, the color represents blue (5ps) directly from trajectory, orange (5ps) from colvars output, green (2ps), red (1ps), purple $(0.5 \mathrm{ps})$, and cyan $(0.2 \mathrm{ps})$. The bold purple is final data for further calculation. $\mathbf{d}$. MFPT plot has the same color representation as PMF plot. The curves with dot solid lines are outward directions of tagged ion and dash curves present inward direction. e. Maximum waiting time between successive transitions. f. Minimum waiting time between successive transitions. g. zposition decorrelation time in each milestoning cell.

\section{MFPT computed from spherical boundary condition}

Laterally confining the ion in the bulk region (cylindrical restraint) is convenient for describing the thermodynamics and kinetics of the ions across the channel along the channel pore axis (z-axis). However, geometries of ion channels are diverse. For funnel shaped the pores (e.g., connexin hemichannel) or pores connected with lateral fenestration (e.g., Piezo1 channel), spherical boundary could be a better choice to capture the distribution and dynamics of ions near the channel entrance. Hence, we further tested milestoning simulation using spherical restraint for ions in the bulk region (Figure 3a). Unlike the cylindrical restraint which yields constant ionic concentration along z-axis, the effective ionic concentration in the spherical bulk cells decrease as the radius of the sphere increase. Thus, the PMF is plotted along the milestoning cell index rather than z-axis. The MFPT is plotted along the milestoning index, which is, in current setup, the edges between cells, excluding the first and last cells.

At low concentration, single channel permeability $\left(\mathrm{cm}^{3} / \mathrm{s}\right)$ can be related linearly to the mean first passage time (MFPT) $\langle t\rangle$ under equilibrium $\mathrm{P}=1 / \mathrm{c}\langle\mathrm{t}\rangle$, in which $\mathrm{c}$ is the symmetric solute concentrations. Since single channel permeability is an intrinsic property of a channel, independent of solute concentration or the shape of the bulk cells, the ratio of MFPT from spherical restraint over cylindrical restraint should equal to the reciprocal of bulk concentration ratio. The concentration of a single ion in a hemisphere with radius of $\sim 9 \AA$ is $1.08 \mathrm{M}$ and in a cylinder with radius of $6 \AA$ and length of $28 \AA$ is $0.52 \mathrm{M}$. Thus, the concentration ratio of 2.07 is 
consistent with the MFPT of $2.6 \mu$ from cylindrical restraint (Figure $2 \mathbf{e}$ ) and $1.3 \mu$ srom spherical restraint (Figure 3c).

a

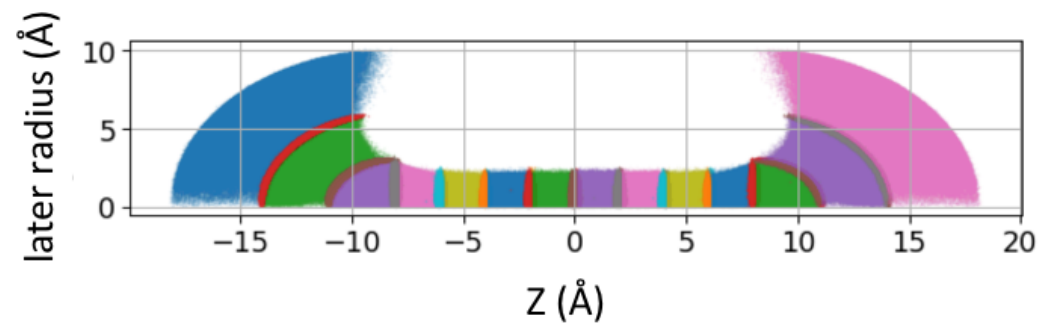

b

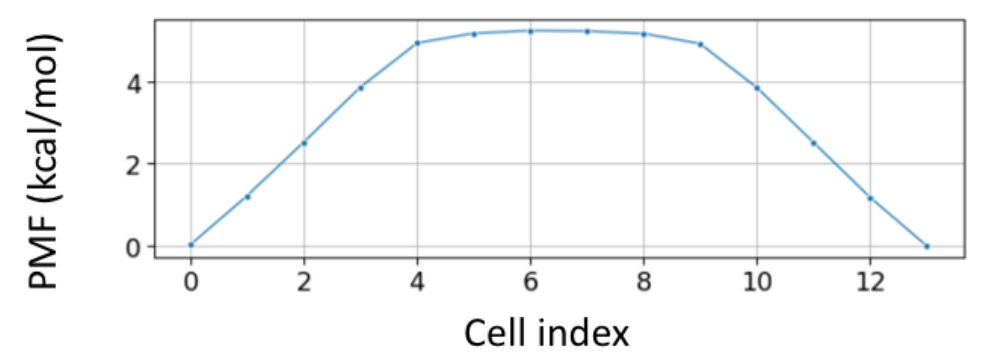

C

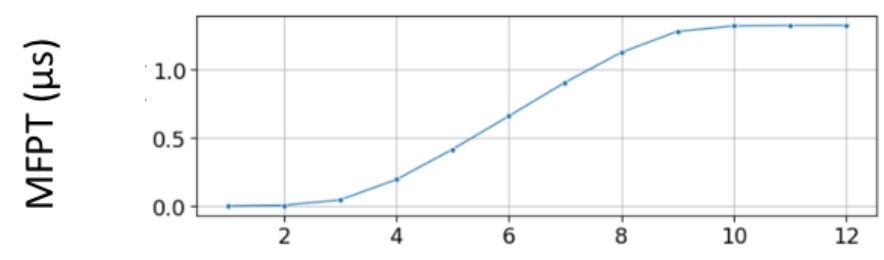

Milestone index

Figure 3. a). Sampling plot with spherical restraint at two bulk ends. The three spherical radius intervals are $3 \AA 3 \AA$ and $4 \AA$ from channel to bulk. b). PMF of spherical restraint system. $X$ axis represents the cell index number. c) MFPT of spherical restraint system. $X$ axis represents the cell index numbers.

\section{MFPT computed from umbrella sampling}

In the high diffusion limit, the MFPT of diffusive motion of $\mathrm{K}^{+}$from the lower to upper boundaries of the channel pore $[z 1, z 2]$ can be written as

$$
M F P T=\int_{Z 1}^{Z 2} \frac{e^{\beta w(Z)}}{D(z)} d z \int_{Z 1}^{Z} e^{-\beta w\left(Z^{\prime}\right)} d z^{\prime} \quad \text { Eq } 5
$$


Eq 5 was originally developed to compute the average reaction time for diffusion processes governed by a Smoluchowski-type diffusion equation ${ }^{38}$. It is also used to derive Eq 4 from Eq $3^{33}$. If we use the PMF and $D(z)$ from umbrella sampling in Eq.5, we obtain MFPT of $2.2 \pm 0.02$ $\mu \mathrm{s}$, quite similar as the $2.6 \mu \mathrm{s}$ MFPT computed directly from milestoning. This consistency further demonstrated the robustness of the current CNT system for computing transport kinetics.

\section{Permeability computed from steady-state flux}

As mentioned above, under low concentration and constant electric field, we can simplify the GHK flux equation for computing the single channel permeability $P$ from conductance simulation. Under a symmetric concentration, GHK flux equation can be simplified to Eq.6.

$$
P=\frac{\gamma R T}{q^{2} F^{2} C}=\frac{\gamma k_{B} T}{q^{2} C} \quad \text { Eq.6 }
$$

where $\gamma$ is the unitary conductance of a single channel, $R$ is the gas constant, $q$ is the charge of the permeating ion, $F$ is the Faraday constant, $C$ is the bulk concentration of the ion, $k_{B} T$ is the same meaning above, except the unit is $\mathrm{eV}$ here $(0.026 \mathrm{eV}$ at $300 \mathrm{~K})$. At sufficiently small $\mathrm{V}$, the current-voltage (I-V) relation is expected to be linear, and the slope defines the conductance.

Ionic conductance from MD simulations can be computed using two approaches. The most commonly used is a direct counting method, in which the currents were computed from the number of permeation events $(N)$ over a simulation period $(\tau), \mathrm{I}=\mathrm{N} / \tau$. In our code, the channel is split into upper, inner, lower regions. A positive permeation event of $\mathrm{K}^{+}$is counted if the time evolution of ion coordinates follows lower-inner-upper sequence, and a negative permeation event is in reverse order. The carbon nanotube is applied with positive and negative $0.4 \mathrm{~V}$ voltage with $200 \mathrm{~ns}$ per replica. Each voltage simulation was repeated three times with different initial velocity. A least-square fitting of the I/V curve gave the conductance of $2.7 \pm 0.94$ pS by direct counting method (Figure 4 a). A more efficient approach that does not rely on completed permeation events is to compute the instantaneous ionic current from charge displacement along z-axis, $I(t)=\sum_{i=1}^{n} q_{i}\left[z_{1}(t+\Delta t)-z_{i}(t)\right] / \Delta t L$, in which qi and zi are the 
charge and $z$ coordinate of ion $i$, and $L$ is the length of the channel pore ${ }^{39}$. This charge displacement method yielded consistent conductance of $2.3 \pm 1.2 \mathrm{pS}$ (Figure $4 \mathbf{b}$ ). With ionic concentration of $0.52 \mathrm{M}$ (one single $\mathrm{K}^{+}$in a cylinder bulk of radius $6 \AA$ and length $28 \AA$ ). The permeability is $(11.9 \pm 6.36) \times 10^{-16} \mathrm{~cm}^{3} / \mathrm{s}$ by charge displacement method, and $(13.6 \pm 4.81) \times 10^{-16}$ $\mathrm{cm}^{3} / \mathrm{s}$ by direct counting method. The above results are calculated by average of the three replicas.

a

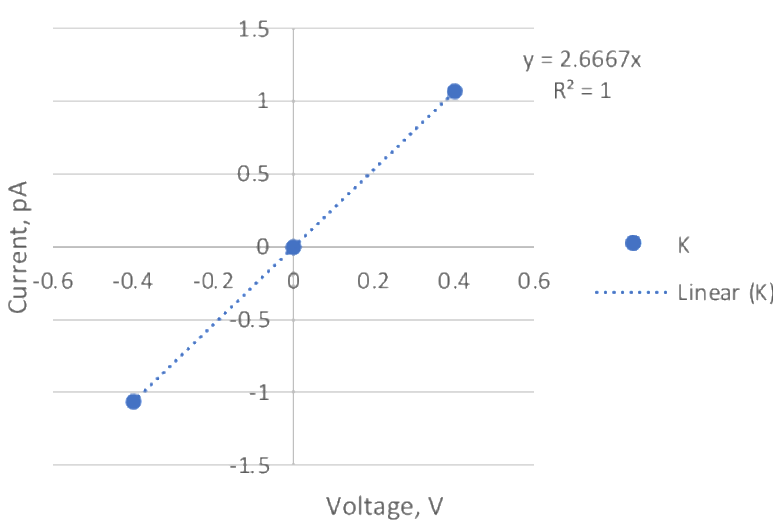

b Conductance-charge displacement

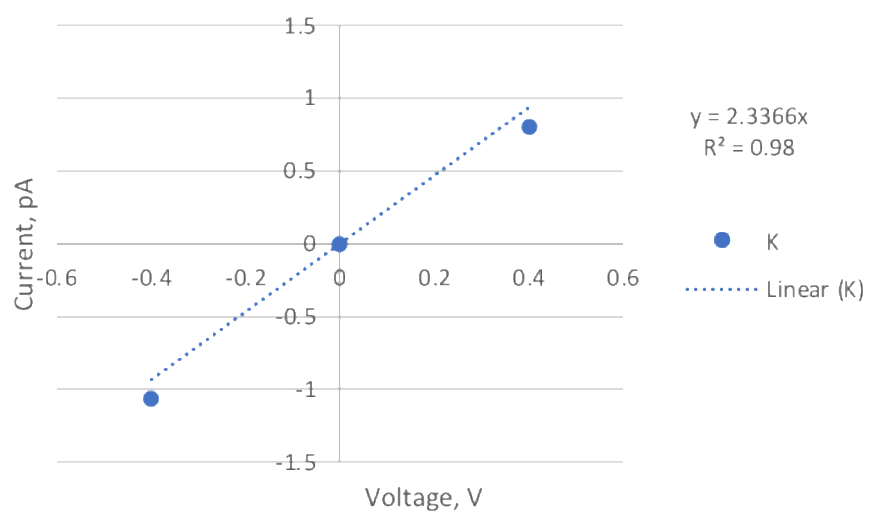

Figure4 a Conductance plot by charge displacement for $+/-0.4 \mathrm{~V}$ which has $6 \AA$ cylinder restraint on a single $\mathrm{K}^{+}$. b. Same voltage trajectory as a but compute by direct counting method.

\section{Conclusion}

In this study, we used a CNT as a toy model of a small conductance ion channel and computed the single channel permeability from umbrella sampling, Markovian milestoning and voltage simulations (Table 1). The PMF from Markovian milestoning method agrees excellently with the PMF from umbrella sampling method. Milestoning simulations were carried out using cylindrical bulk restraint and spherical bulk restraint. Both boundary conditions yield consistent MFPT for a single $\mathrm{K}^{+}$permeates through a carbon nanotube without external voltage. Despite the distinct underlying theories and simulation approaches, all three methods generated consistent single 
channel permeability. These results are also in the same range as the previously reported $\mathrm{K}^{+}$ permeability of $(25 \pm 7) \times 10^{-16} \mathrm{~cm}^{3} / \mathrm{s}$ computed using a transition path approach with CHARMM22 force fied ${ }^{17}$.

In terms of computational resources, umbrella sampling has the advantage because PMF converges much faster than MFPT. However, MFPT allows extract the kinetics directly from sampling, thus does not rely on the assumption of ISD formulism. In fact, for most ion channels that have multiple free energy barriers, MFPT provide detailed kinetics at each position inside the pore. Steady-state flux is straight forward to apply if the permeant is charged and sufficient sampling is achievable under reasonable voltages. However, if the permeant is not charged, a constant concentration gradient needs to be applied. Furthermore, the effect of an unphysiologically large voltage bias or electrochemical gradient on channel property is likely system dependent and difficult to predict over a long simulation time. Compared with the steadystate flux approaches, milestoning approach does not depend on the charge of the permeant, thus can be used to study any types of small molecular permeation. Therefore, the use of milestoning has a significant promise for future applications for complex systems that are hard to extract kinetics from unbiased MD or PMF-based enhanced sampling approaches.

Table 1. Summary of the three methods for computing single channel permeability

\begin{tabular}{|c|c|c|c|}
\hline Method & Umbrella sampling & Markovian milestoning & Steady-state flux \\
\hline Permeability equation & $P=\pi r^{2}\left(\int_{z_{1}}^{z_{2}} \frac{e^{w(z) / k_{B} T}}{D(z)} d z\right)$ & $P=\frac{\pi r^{2} \int_{Z 1}^{Z 2} e^{-w(Z) / k_{B} T} d z}{2 \times M F P T}$ & $P=\frac{\gamma k_{B} T}{q^{2} C}$ \\
\hline Effective bulk conc. & $0.77 \mathrm{M}$ & $0.77 \mathrm{M}$ & $0.52 \mathrm{M}$ \\
\hline Biasing potential & Harmonic & Flat-bottom harmonic & Voltage $\pm 0.4 \mathrm{~V}$ \\
\hline Biasing force constant & $2.5 \mathrm{kcal} / \mathrm{mol}$ & $100 \mathrm{kcal} / \mathrm{mol}$ & - \\
\hline Lateral restraint in bulk & $6 \AA$ & $6 \AA$ & - \\
\hline PMF barrier (kcal/mol) & $3.8 \pm 0.17$ & $4.1 \pm 1.7$ & - \\
\hline Key parameters & $\mathrm{D}(\mathrm{z}) 0.004 \sim 0.028 \AA^{2} / \mathrm{ps}$ & MFPT: $2.6 \mu \mathrm{s}$ & conductance $2.3 \pm 1.2 \mathrm{pS}$ \\
\hline Permeability $\left(\mathrm{cm}^{3} / \mathrm{s}\right)$ & $(8.96 \pm 0.02) \times 10^{-16}$ & $2.92 \times 10^{-16}$ & $(11.9 \pm 6.36) \times 10^{-16}$ \\
\hline Total sampling time ( $\mu \mathrm{s})$ & 1.12 & 4.98 & 0.60 \\
\hline
\end{tabular}




\section{Acknowledgment}

We would like to thank Dr. Fangqiang Zhu for sharing the coordinates of the CNT model used for this study. Computational resources were provided via the Extreme Science and Engineering Discovery Environment (XSEDE) allocation TG-MCB160119, which is supported by NSF grant number ACI-154862.

\section{References}

1. Fowler, P. W.; Abad, E.; Beckstein, O.; Sansom, M. S., Energetics of Multi-Ion Conduction Pathways in Potassium Ion Channels. J Chem Theory Comput 2013, 9, 5176-5189.

2. Allen, T. W.; Andersen, O. S.; Roux, B., Energetics of Ion Conduction through the Gramicidin Channel. Proc Natl Acad Sci U S A 2004, 101, 117-122.

3. Berneche, S.; Roux, B., Energetics of Ion Conduction through the K+ Channel. Nature 2001, 414, 73-77.

4. Domene, C.; Klein, M. L.; Branduardi, D.; Gervasio, F. L.; Parrinello, M., Conformational Changes and Gating at the Selectivity Filter of Potassium Channels. J Am Chem Soc 2008, 130, 9474-9480.

5. Khalili-Araghi, F.; Tajkhorshid, E.; Schulten, K., Dynamics of K+ Ion Conduction through Kv1.2. Biophys J 2006, 91, L2-L4.

6. Roux, B., The Membrane Potential and Its Representation by a Constant Electric Field in Computer Simulations. Biophys J 2008, 95, 4205-4216.

7. Kutzner, C.; Grubmuller, H.; de Groot, B. L.; Zachariae, U., Computational Electrophysiology: The Molecular Dynamics of Ion Channel Permeation and Selectivity in Atomistic Detail. Biophys J 2011, 101, 809-817.

8. Khalili-Araghi, F.; Ziervogel, B.; Gumbart, J. C.; Roux, B., Molecular Dynamics Simulations of Membrane Proteins under Asymmetric Ionic Concentrations. J Gen Physiol 2013, 142, 465-475.

9. Adelman, J. L.; Grabe, M., Simulating Current-Voltage Relationships for a Narrow Ion Channel Using the Weighted Ensemble Method. J Chem Theory Comput 2015, 11, 1907-1918.

10. Domene, C.; Ocello, R.; Masetti, M.; Furini, S., Ion Conduction Mechanism as a Fingerprint of Potassium Channels. J Am Chem Soc 2021, 143, 12181-12193.

11. Hempel, T.; del Razo, M. J.; Lee, C. T.; Taylor, B. C.; Amaro, R. E.; Noe, F., Independent Markov Decomposition: Toward Modeling Kinetics of Biomolecular Complexes. P Natl Acad Sci USA 2021, 118.

12. Teo, I.; Schulten, K., A Computational Kinetic Model of Diffusion for Molecular Systems. Journal of Chemical Physics 2013, 139.

13. Choudhary, O. P.; Paz, A.; Adelman, J. L.; Colletier, J. P.; Abramson, J.; Grabe, M., Structure-Guided Simulations Illuminate the Mechanism of Atp Transport through Vdac1. Nat Struct Mol Biol 2014, 21, 626632.

14. Jiang, W. J.; Lin, Y. C.; Botello-Smith, W.; Contreras, J. E.; Harris, A. L.; Maragliano, L.; Luo, Y. L., Free Energy and Kinetics of Camp Permeation through Connexin26 Via Applied Voltage and Milestoning. Biophys J 2021, 120, 2969-2983.

15. Cottone, G.; Chiodo, L.; Maragliano, L., Thermodynamics and Kinetics of Ion Permeation in WildType and Mutated Open Active Conformation of the Human Alpha 7 Nicotinic Receptor. J Chem Inf Model 2020, 60, 5045-5056.

16. Alberini, G.; Benfenati, F.; Maragliano, L., Molecular Dynamics Simulations of Ion Selectivity in a Claudin-15 Paracellular Channel. J Phys Chem B 2018, 122, 10783-10792. 
17. Zhou, X. Y.; Zhu, F. Q., Calculating Single-Channel Permeability and Conductance from Transition Paths. J Chem Inf Model 2019, 59, 777-785.

18. Faradjian, A. K.; Elber, R., Computing Time Scales from Reaction Coordinates by Milestoning. $J$ Chem Phys 2004, 120, 10880-10889.

19. Maragliano, L.; Vanden-Eijnden, E.; Roux, B., Free Energy and Kinetics of Conformational Transitions from Voronoi Tessellated Milestoning with Restraining Potentials. J Chem Theory Comput 2009, 5, 2589-2594.

20. MacKerell Jr, A. D.; Bashford, D.; Bellott, M.; Dunbrack Jr, R. L.; Evanseck, J. D.; Field, M. J.; Fischer, S.; Gao, J.; Guo, H.; Ha, S.; Joseph-McCarthy, D.; Kuchnir, L.; Kuczera, K.; Lau, T. K.; Mattos, C.; Michnick, S.; Ngo, T.; Nguyen, D. T.; Stote, R.; Straub, J.; Watanabe, M.; Wiorkiewicz-Kuczera, J.; Yin, D.; Karplus, M., All-Atom Empirical Potential for Molecular Modeling and Dynamics Studies of Proteins. J Phys Chem B 1998, 102, 3586-3616.

21. Mackerell Jr, A. D.; Feig, M.; Brooks III, C. L., Extending the Treatment of Backbone Energetics in Protein Force Fields: Limitations of Gas-Phase Quantum Mechanics in Reproducing Protein Conformational Distributions in Molecular Dynamics Simulations. J Comput Chem 2004, 25, 1400-1415.

22. Hoover, W. G.; Ladd, A. J.; Moran, B., High-Strain-Rate Plastic Flow Studied Via Nonequilibrium Molecular Dynamics. Phys Rev Lett 1982, 48, 1818.

23. Evans, D. J., Computer "Experiment"for Nonlinear Thermodynamics of Couette Flow. J Chem Phys 1983, 78, 3297-3302.

24. Darden, T.; York, D.; Pedersen, L., Particle Mesh Ewald: An N. Log (N) Method for Ewald Sums in Large Systems. J Chem Phys 1993, 98, 10089-10092.

25. Shirts, M. R.; Chodera, J. D., Statistically Optimal Analysis of Samples from Multiple Equilibrium States. The Journal of chemical physics 2008, 129, 124105.

26. Chodera, J. D.; Swope, W. C.; Pitera, J. W.; Seok, C.; Dill, K. A., Use of the Weighted Histogram Analysis Method for the Analysis of Simulated and Parallel Tempering Simulations. Journal of Chemical Theory and Computation 2007, 3, 26-41.

27. Hummer, G., Position-Dependent Diffusion Coefficients and Free Energies from Bayesian Analysis of Equilibrium and Replica Molecular Dynamics Simulations. New J Phys 2005, 7.

28. Woolf, T. B.; Roux, B., Conformational Flexibility of O-Phosphorylcholine and OPhosphorylethanolamine - a Molecular-Dynamics Study of Solvation Effects. J Am Chem Soc 1994, 116, 5916-5926.

29. Berne, B. J.; Borkovec, M.; Straub, J. E., Classical and Modern Methods in Reaction-Rate Theory. J Phys Chem-Us 1988, 92, 3711-3725.

30. Diamond, J. M.; Katz, Y., Interpretation of Nonelectrolyte Partition-Coefficients between Dimyristoyl Lecithin and Water. J Membrane Biol 1974, 17, 121-154.

31. Venable, R. M.; Kramer, A.; Pastor, R. W., Molecular Dynamics Simulations of Membrane Permeability. Chem Rev 2019, 119, 5954-5997.

32. Awoonor-Williams, E.; Rowley, C. N., Molecular Simulation of Nonfacilitated Membrane Permeation. Bba-Biomembranes 2016, 1858, 1672-1687.

33. Votapka, L. W.; Lee, C. T.; Amaro, R. E., Two Relations to Estimate Membrane Permeability Using Milestoning. J Phys Chem B 2016, 120, 8606-8616.

34. Zhu, F. Q.; Hummer, G., Theory and Simulation of Ion Conduction in the Pentameric Glic Channel. Journal of Chemical Theory and Computation 2012, 8, 3759-3768.

35. Allen, T. W.; Andersen, O. S.; Roux, B., Energetics of Ion Conduction through the Gramicidin Channel. P Natl Acad Sci USA 2004, 101, 117-122.

36. Vanden-Eijnden, E.; Venturoli, M.; Ciccotti, G.; Elber, R., On the Assumptions Underlying Milestoning. J Chem Phys 2008, 129, 174102. 
bioRxiv preprint doi: https://doi.org/10.1101/2021.11.20 469392; this version posted November 20, 2021. The copyright holder for this preprint (which was not certified by peer review) is the author/funder, who has granted bioRxiv a license to display the preprint in perpetuity. It is made available under aCC-BY-ND 4.0 International license.

37. Vanden-Eijnden, E.; Venturoli, M., Markovian Milestoning with Voronoi Tessellations. J Chem Phys 2009, 130, 194101.

38. Szabo, A.; Schulten, K.; Schulten, Z., First Passage Time Approach to Diffusion Controlled Reactions. The Journal of Chemical Physics 1980, 72, 4350-4357.

39. Aksimentiev, A.; Schulten, K., Imaging Alpha-Hemolysin with Molecular Dynamics: Ionic Conductance, Osmotic Permeability, and the Electrostatic Potential Map. Biophys J 2005, 88, 3745-3761. 